\title{
Adaptive Nonsingular Terminal Sliding Model Control and Its Application to Permanent Magnet Synchronous Motor Drive System
}

\author{
Yue Liu ${ }^{1}$, Shuo Zhou ${ }^{2}$ \\ ${ }^{1}$ School of Science, Shenyang Jianzhu University, Shenyang 110168, China \\ ${ }^{2}$ Department of Mechanical and Electronic Engineering, Guidaojiaotong Polytechnic Institute, Shenyang 110023, China
}

\begin{abstract}
To improve the dynamic performance of permanent magnet synchronous motor(PMSM) drive system, a adaptive nonsingular terminal sliding model control((NTSMC) strategy was proposed. The proposed control strategy presents an adaptive variable-rated exponential reaching law which the L1 norm of state variables is introduced. Exponential and constant approach speed can adaptively adjust according to the state variables' distance to the equilibrium position. The proposed scheme can shorten the reaching time and weaken system chatting. The method was applied to the PMSM speed servo system, and compared with the traditional terminal-sliding-mode regulator and PI regulator. Simulation results show that the proposed control strategy can improve dynamic, steady performance and robustness.
\end{abstract}

\section{Introduction}

Permanent magnetic synchronous motors have widely been used in AC driving system due to the high efficiency, high power density and good controllability. However, it is still a challenging problem to control the PMSM to get the perfect dynamic performance, because the dynamic model of PMSM is multivariable, strong coupling, nonlinear and parameters variable [1-5]. So the study on speed control of PMSM has attracted considerable attention among the scholars. Many different control methods have been proposed such as adaptive control, robust control, backstepping method, direct torque control, fuzzy logic control, etc [6-10].

In recent years, many scholars have devoted their research to applying sliding mode variable structure control (SMC) to PMSMs because SMC has good robustness to parameter perturbations, external disturbances and inaccurate mathematical description [11-15]. In [11], a variable parameter SMC was studied using reaching law approach. This method can improve dynamic and static performances, but chattering problem is not suppressed effective. A neuron-fuzzy sliding-mode controller was reported for PMSM control system in [12]. This method weakens the effects of switching control and chattering. In [13-14] a sliding mode observer (SMO) was proposed to estimate rotor position and velocity of PMSM using current and voltage of stator. But due to the presence of chattering, the output of the SMO needs to be passed through the LPF, which causes a time delay and requires extra compensation for the rotor position estimation. In [15] a terminal sliding mode controller of PMSM was designed for alleviating the chattering.
However, the convergence rate and dynamic performance are not satisfied.

In this paper, an adaptive variable-rated exponential reaching law is proposed, where the L1 norm of state variables is introduced. In order to shorten the reaching time, exponential approach speed can be adjusted according to the state variables' distance to the equilibrium position. Constant approach speed can also be adaptively adjusted, which can weaken the system chattering. In order to verify the validity of proposed control strategy in PMSM, control effect will be compared with traditional terminal sliding mode method and PI regulator. Simulation and experimental results show that the proposed control strategy can improve dynamic,steady performance and robustness.

\section{Performance analysis of adaptive nonsingular terminal sliding model control}

Consider the following second-order nonlinear dynamic systems:

$$
\left\{\begin{array}{l}
\dot{x}_{1}=x_{2} \\
\dot{x}_{2}=f(x)+b(x) u+g(t)
\end{array}\right.
$$

where $\quad x=\left[x_{1}, x_{2}\right]^{T} \in R^{2} \quad$ are state variables. $b(x) \neq 0 ; g(t)$ is an uncertain term, the uncertain part is assumed to be bounded as $|g(t)| \leq l, l$ is constant number; 
$u$ is input of system.For system (1), the conventional exponential reaching law is as follow:

$$
\dot{s}=-\varepsilon \operatorname{sgn}(s)-k s, k>0, \varepsilon>0
$$

The reaching law contains two parts. One is the exponential approach $\dot{S}=-k s$. Another is constant approach $\dot{s}=-\varepsilon \operatorname{sgn}(s)$. In equation (2), the coefficient $k, \varepsilon$ do not have adaptive function. The convergence properties cannot achieve the best performance for different position state variables.In this paper, an adaptive variable-rated exponential reaching law is proposed as:

$$
\dot{s}=-\varepsilon \frac{1}{1+c\|x\|_{1}} \operatorname{sgn}(s)-\left(k+c\|x\|_{1}\right) s
$$

where $\|x\|_{1}=\sum_{i=1}^{n} x_{i}$ is the $L_{1}$ norm of state variables. $k>0, \varepsilon>0, c>0, n>0$.

The exponential and constant approach speed can adaptively adjust according to the state variables' distance to the equilibrium position. In equation (3), the transient solution of exponential approach is $s=s(0) e^{-\left(k+c\|x\|_{1}\right)^{t}}$. The proposed exponential decay rate is faster than one's in equation (2) when $\|x\|_{1}$ is large. The reaching time can be shortened. The constant reaching law is $\varepsilon \frac{1}{1+c\|x\|_{1}}$, which is much smaller than $\varepsilon$ in equation (2). The reaching time can also be shortened by adjusting coefficient c when $\|x\|_{1}$ is small. At the same time the system chattering can be weakened. According to the nonlinear dynamic systems (1), a terminal sliding surface is proposed as:

$$
s=x_{1}+\frac{1}{\beta} x_{2}^{p / q}
$$

The control law is designed as follow:

$$
\begin{aligned}
& u=-b^{-1}(x)\left[f(x)+\beta \frac{q}{p} x_{2}{ }^{2-\frac{p}{q}}+l \operatorname{sgn}(s)+\right. \\
& \left.\varepsilon \frac{1}{1+c\|x\|_{1}} \operatorname{sgn}(s)+\left(k+c\|x\|_{1}\right) s\right]
\end{aligned}
$$

where $p, q \in N$ are the odd number, $1<p / q<2$. $\beta>0, l>0, k>0, \varepsilon>0, c>0$.

In order to prove the stability of the scheme presented in (5), the Lyapunov function candidate $\mathrm{V}$ is defined as

$$
V=\frac{1}{2} s^{2}
$$

The time derivative of (6) is given by

$$
\begin{gathered}
\dot{V}=s \dot{s}=s\left(x_{2}+\frac{1}{\beta} \frac{p}{q} x_{2}{ }^{\frac{p}{q}-1} \dot{x}_{2}\right)= \\
\frac{1}{\beta} \frac{p}{q} x_{2}{ }^{\frac{p}{q}-1}\left\{s[g(t)-l \operatorname{sgn}(s)]-\varepsilon \frac{1}{1+c\|x\|_{1}}|s|-\right. \\
\left.\left(k+c\|x\|_{1} s^{2}\right)\right\} \leq \frac{1}{\beta} \frac{p}{q} x_{2}^{\frac{p}{q}-1}\left[-\varepsilon \frac{1}{1+c\|x\|_{1}}|s|-\right. \\
\left.\left(k+c\|x\|_{1}\right) s^{2}\right] \leq 0
\end{gathered}
$$

Therefore, the reaching condition $s \dot{s}<0$ will be satisfied. The system can converge to the equilibrium in finite time.

In order to analysis performance of proposed controller, taking (7) as a typical system expressed as follows:

$$
\left\{\begin{array}{l}
\dot{x}_{1}=x_{2} \\
\dot{x}_{2}=-25 x_{2}+133 u+0.1 \sin (20 t)
\end{array}\right.
$$

The initial condition of system is $x^{\mathrm{T}}(0)=[10,10]^{\mathrm{T}}$. The result of comparison between proposed control method and traditional terminal sliding mode regulator is shown in Fig. 1.The convergence speed of proposed method is faster significantly than the traditional terminal sliding mode regulator's.

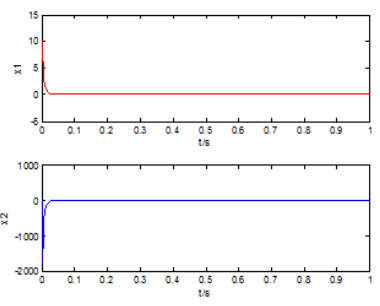

(a) Process of status convergence (Proposed method)

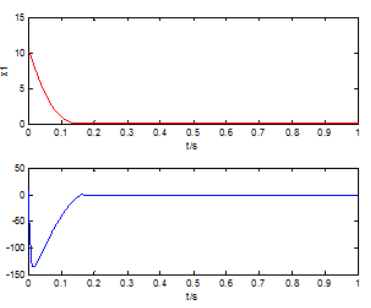

(b) Process of status convergence ( Traditional terminal sliding mode

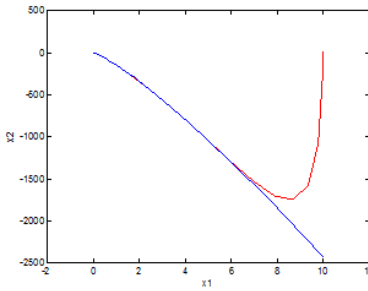

(c) Phase trajectory (Proposed method)

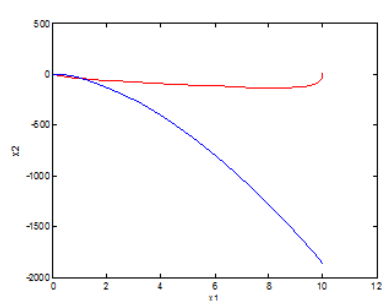

(d) Phase trajectory

(Traditional terminal sliding mode method)
Figure 1. Performance comparisons of proposed method and traditional terminal sliding mode method.

\section{Application of adaptive nonsingular terminal sliding model control method on PMSM}

\subsection{PMSM modeling}

Assume that magnetic circuit is unsaturated, hysteresis and eddy current loss are ignored and the distribution of 
the magnetic field is sine space. In d-q coordinates the model of PMSM can be expressed as follows:

$$
\left\{\begin{array}{l}
u_{d}=r i_{d}-\omega L_{q} i_{q}+\frac{d \psi_{d}}{d t} \\
u_{q}=r i_{q}+\omega L_{d} i_{d}+\frac{d \psi_{q}}{d t} \\
T_{e}=\frac{3}{2} p \psi_{f} i_{q} \\
T_{e}-T_{L}=\frac{J}{p} \frac{d \omega}{d t}
\end{array}\right.
$$

Flux equations are as follows:

$$
\left\{\begin{array}{l}
\psi_{d}=L_{d} i_{d}+\psi_{f} \\
\psi_{q}=L_{q} i_{q}
\end{array}\right.
$$

where $u_{d}, u_{q}$ represent $d$ and $q$ axes stator voltages respectively; $i_{d}, i_{q}$ are $d$ and $q$ axes currents respectively; $L_{d}, L_{q}$ are $d$ and $q$ axes stator inductance; $r$ is stator resistance; $p$ is number of pole pairs; $\omega$ is electrical angular velocity; $J$ is rotational inertia; $T_{e}$ is electrical magnetic torque; $T_{L}$ is load torque; $\psi_{f}$ permanent magnet flux.

\subsection{Design of NTSMC speed controller}

State variables of PMSM are as follows:

$$
\left\{\begin{array}{l}
x_{1}=\omega^{*}-\omega \\
x_{2}=\dot{x}_{1}=-\dot{\omega}
\end{array}\right.
$$

where $\omega^{*}$ is reference velocity, $\omega$ is actual velocity. According to (8) and (10), state variables can be described as follows:

$$
\left\{\begin{array}{l}
\frac{d x_{1}}{d t}=-\frac{d \omega}{d t}=-\frac{p}{J}\left(\frac{3}{2} p \psi_{f} i_{q}-T_{L}\right) \\
\frac{d x_{2}}{d t}=-\frac{d^{2} \omega}{d t^{2}}=-\frac{3 p^{2}}{2 J} \psi_{f} \frac{d i_{q}}{d t}
\end{array}\right.
$$

Considering the uncertain term $g_{1}(t)$ of PMSM, the differential equation (11) is rewritten as

$$
\begin{aligned}
& \int \frac{d x_{1}}{d t}=-\frac{d \omega}{d t}=-\frac{p}{J}\left(\frac{3}{2} p \psi_{f} i_{q}-T_{L}\right) \\
& \frac{d x 2}{d t}=-\frac{d^{2} \omega}{d t^{2}}=-\frac{3 p^{2}}{2 J} \psi_{f} \frac{d i_{q}}{d t}+g_{1}(t) \\
& \Rightarrow\left\{\begin{array}{l}
\dot{x}_{1}=x_{2} \\
\dot{x}_{2}=-b u+g_{1}(t)
\end{array}\right.
\end{aligned}
$$

where $b=\frac{3 p^{2}}{2 J} \psi_{f}, u=\frac{d i_{q}}{d t},\left|g_{1}(t)\right| \leq l_{1}$.

The sliding surface is considered as

$$
s_{1}=x_{1}+\frac{1}{\beta_{1}} x_{2}^{p_{1} / q_{1}}
$$

The control input $i_{q}$ is designed as follows:

$$
\begin{aligned}
i_{q}= & \frac{2 J}{3 p^{2} \psi_{f}} \int_{0}^{t} \beta_{1} \frac{q_{1}}{p_{1}} x_{2}{ }^{2-\frac{p_{1}}{q_{1}}}+l_{1} \operatorname{sgn}\left(s_{1}\right)+ \\
& \left.\varepsilon_{1} \frac{1}{1+c_{1}\|x\|_{1}} \operatorname{sgn}\left(s_{1}\right)+\left(k_{1}+c_{1}\|x\|_{1}\right) s_{1}\right] d t
\end{aligned}
$$

The block diagram of PMSM speed-regulation system is shown in Fig. 2.

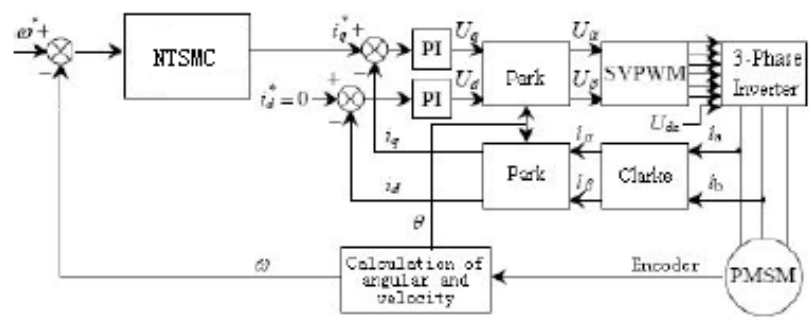

Figure 2. The block diagram of PMSM speed-regulation system.

\section{Simulation results}

To demonstrate the effectiveness of the proposed approach, simulations of proposed method, the PI method and the traditional terminal sliding mode method in one PMSM system were made. The related parameters of PMSM are shown in Table 1.

Table 1. Parameters of PMSM

\begin{tabular}{|c|c|}
\hline Parameter & Value \\
\hline Stator Resistance $(r)$ & $2.46 \Omega$ \\
\hline Stator Inductor $\left(L_{d}, L_{q}\right)$ & $6.35 \mathrm{mH}$ \\
\hline Flux $\left(\psi_{f}\right)$ & $0.175 \mathrm{~Wb}$ \\
\hline Rotor Inertia $(J)$ & $1.02 \mathrm{e}-3 \mathrm{~kg} \cdot \mathrm{m} 2$ \\
\hline Poles $(p)$ & 4 \\
\hline Rated speed $\left(\omega^{*}\right)$ & $3000 \mathrm{r} / \mathrm{min}$ \\
\hline
\end{tabular}

The comparison curve of speed response among adaptive nonsingular terminal sliding model regulator, PI regulator and traditional terminal sliding mode regulator are showed in Fig. 3. From the simulation results, it can be observed that PI controller has the fluctuation and overshoot obviously. The response time of traditional terminal sliding mode controller is longer than proposed 
controller. The response curve of speed, load torque and current are be shown in Fig. 4 (a)-(c) when load torque is added suddenly form $1 \mathrm{~N} \cdot \mathrm{m}$ to $7 \mathrm{~N} \cdot \mathrm{m}$ at $t=0.05 \mathrm{~s}$.It can be observed that the proposed method has a smaller speed fluctuation and a shorter settling time compared with the PI method when the load torque changes. Moreover, the dynamic responses of speed and current using PI method are much sensitive to loading.

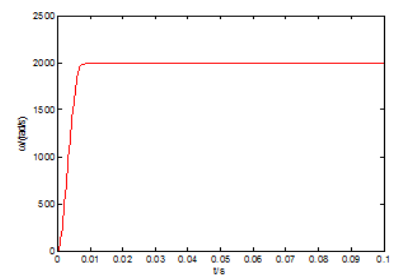

(a) Proposed method

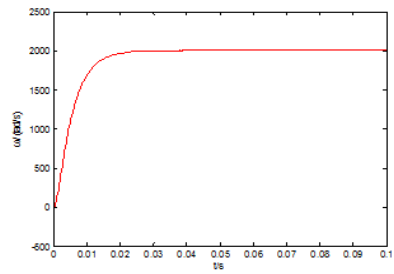

(c) Traditional terminal sliding mode method

Figure 3. Speed response when system starting

\section{Conclusion}

In this paper, an adaptive variable-rated exponential reaching law is presented which the L1 norm of state variables is introduced. Exponential and constant approach speed can adaptively adjust according to the state variables' distance to the equilibrium position. The proposed scheme can shorten the reaching time and weaken system chatting. The simulation results demonstrate the effectiveness of the proposed method.

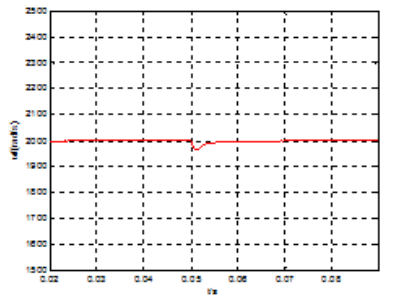

Proposed method

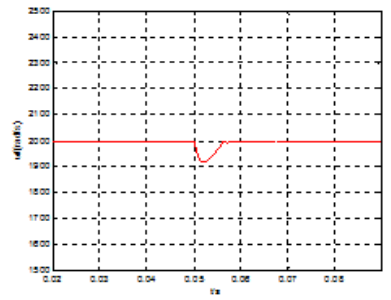

PI method (a) Speed response adding load suddenly

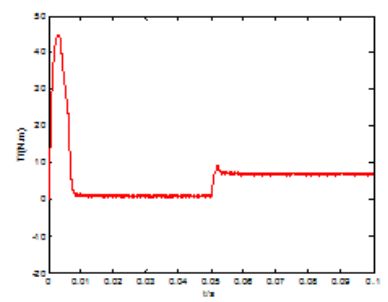

Proposed method

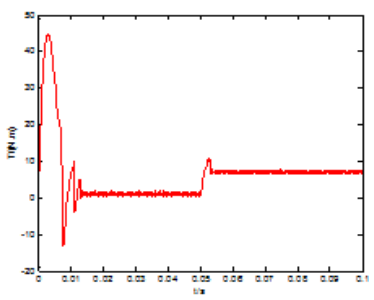

PI method
(b)Torque response adding load suddenly

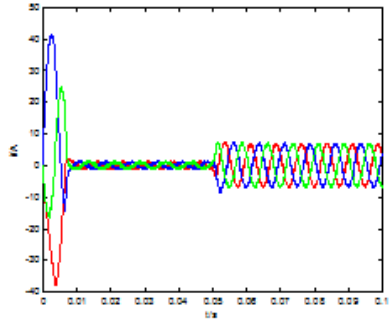

Proposed method

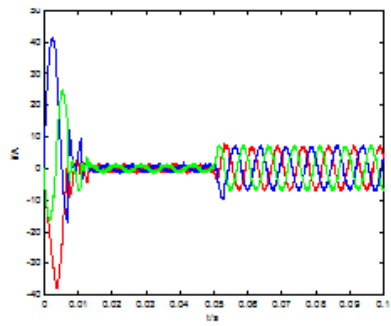

PI method
(c)Current response adding load suddenly

Figure 4. Simulation results of system dynamic response to loading

\section{References}

1. Sun Qiang, Cheng Ming, Zhou Er, Proceedings of the CSEE, 23,6 (2003)

2. Wang Jiang, Wang Jing,Fei Xiangyang, Proceedings of the CSEE. 25, 7, (2005)

3. Uddin M N,Abido M A,Rahman M A, IEEE Transactions on Industry Applications, 40, 1, (2004)

4. WANG Baoren,ZHANG Chengrui,JIA Lei, Electric Machines and Control,11,3, (2007)

5. Miller C E, Van Zyl A W, Landy C F, IEEE Africon Conference (2002)

6. QIU Jun,ZHAO Guang-zhou, Electric Machines and Control, 13, 6(2009)

7. YANG Shu-sheng, ZHONG Yi-sheng, Proceedings of the CSEE, 29,3(2009)

8. ZHOU Guo-rong, LI Yong-feng, Control Engineering of China,16,1(2009)

9. Yongchang Zhang,Jianguo Zhu, IEEE Transactions on Power Electronics,26,1(2011)

10. Stone Cheng,Chi-Wei Li, Control Engineering Practice, 19,4(2011)

11. Wang Haibo,Zhou Bo,Fang Si-chen, Transactions of China Electrotechnical Society, 24,9(2009)

12. Cetin Elmas, Oguz Ustun, Control Engineering Practice, 16,6(2008)

13. SHANG Zhe, ZHAO Rong-xiang, DOU Re-zhen, Proceedings of the CSEE, 27,3(2007)

14. Liu Jun, Wang Gang, Yu JinShou, Proceedings of the 8th World Congress on Intelligent Control and Automation, (2010)

15. WANG Yan-min, FENG Yong, LU Qi-liang, Electric Machines and Control, 12, 5(2008) 